CGPG-96/1-1

gr-qc/9601009

\title{
Analytic approximations to the spacetime of a critical gravitational collapse
}

\author{
Richard H. Price \\ Department of Physics, University of Utah \\ Salt Lake City, UT 84112 \\ Jorge Pullin \\ Center for Gravitational Physics and Geometry, Department of Physics \\ 104 Davey Lab, The Pennsylvania State University, University Park, PA 16802
}

\begin{abstract}
We present analytic expressions that approximate the behavior of the spacetime of a collapsing spherically symmetric scalar field in the critical regime first discovered by Choptuik. We find that the critical region of spacetime can usefully be divided into a "quiescent" region and an "oscillatory" region, and a moving "transition edge" that separates the two regions. We find that in each region the critical solution can be well approximated by a flat spacetime scalar field solution. A qualitative nonlinear matching of the solutions across the edge yields the right order of magnitude for the oscillations of the discretely self-similar critical solution found by Choptuik.
\end{abstract}

\section{INTRODUCTION}

The dynamics of a spherically-symmetric massless scalar field coupled to general relativity can have two late-time outcomes. Either a black hole forms or the scalar field energy eventually radiates away, leaving spacetime flat. By studying solutions very close to the critical case (formation of a black hole of vanishing mass) Choptuik [1] has found two very interesting phenomena, as yet not explained in any fundamental way: universality and echoing. Similarities to the Choptuik results have been found in other contexts. Abrahams and Evans [2] studied the collapse of axisymmetric vacuum gravitational fields. Coleman and Evans 33 have studied the collapse of spherically symmetric radiative fluids, and Eardley and Hirshmann 4 studied the collapse of spherically symmetric complex scalar fields.

"Universality" is a feature of supercritical cases, those in which holes form. The dependence of the mass of the final hole on a parameter of the initial data, appears to have a universal power-law character and this character, as well as the numerical value of the exponent appear to be the same for different families of data, though not, as originally thought, for different collapsing fields [5,6]. This feature of gravitational collapse would seem, therefore, to have the same universal character - as well as the same mathematical appearance - as phase transitions in statistical mechanics.

In sharp contrast, "echoing" defies close comparison with any other physical phenomenon. For solutions very close to criticality, the evolving fields go through a sequence of cycles, each of which duplicates the previous one, but on a radial scale smaller by around a factor of 30, and on a time scale smaller by the same factor. This has been seen only in the work of Choptuik [1] and Abrahams and Evans [2] axially symmetric examples, in which the echoing occurred with a scaling factor around 6 , rather than 30 as in Choptuik's spherically symmetric results. Very recently Gundlach [7], with an independent numerical approach, has confirmed that the echoing is an exact feature of the solution. For fluid collapse [3] and the complex scalar field [4] exact self-similar critical solutions are known, with no echoing (in the complex scalar field case there is echoing in the phase). It is widely suspected that the echoing behavior has an origin related to self-similarity, but a detailed understanding is still lacking.

In this paper we will consider only that feature of Choptuik's solution which we consider most unusual, and surely that which is least understood: the echoing. Since no "simple" model problem is yet know which has this feature, we focus instead on the equations of Choptuik's physical problem to see the manner in which the echoing solution arises. We are, then, in some sense dealing only with the "how" of the solution, rather than the "why," in the hope that this might be a useful step.

With this aim we will divide the spacetime into two regions where we are able to find approximate solutions to the coupled Einstein-Scalar field equations and we will discuss a nonlinear matching 
between them. It wil turn out, somewhat surprisingly, that in the two relevant regions, the behavior is mainly dominated by flat spacetime evolutions. The oscillatory behavior, which $a b$ initio appears as a feature of the nonlinearities of general relativity, can actually be accounted for by flat spacetime behavior. The nonlinear nature of the theory is necessary to join the solution in the two regions, and this join determines the scaling factor of the echoing.

The plan of this paper is as follows. In section II we present the basic equations and discuss the results for the dynamics observed by Choptuik. In section III we present our approximate solutions and discuss their regions of validity. In section IV we discuss the relevance of these approximate solutions.

\section{BASIC EQUATIONS AND CHOPTUIK'S SOLUTION}

The physical problem is the dynamics of a scalar field $\phi$ coupled to general relativity. The combined set of Einstein-Scalar equations are,

$$
\begin{aligned}
R_{\mu \nu} & =8 \pi \partial_{\mu} \phi \partial_{\nu} \phi \\
\square \phi & =0 .
\end{aligned}
$$

We assume spherical symmetry for the scalar field and the spacetime, so the metric can be written as

$$
d s^{2}=-\alpha^{2}(r, t) d t^{2}+a^{2}(r, t) d r^{2}+r^{2}\left(d \theta^{2}+\sin ^{2} \theta d \varphi^{2}\right) .
$$

It is useful to introduce the mass function

$$
m(r, t)=r \frac{1-a^{-2}(r, t)}{2} .
$$

and the dimensionless "phase space" variables used by Choptuik:

$$
X(r, t)=\sqrt{2 \pi} \frac{r}{a} \frac{\partial \phi}{\partial r}, \quad Y(r, t)=\sqrt{2 \pi} \frac{r}{\alpha} \frac{\partial \phi}{\partial t} .
$$

With these choices, the Einstein-scalar equations reduce to a set of four partial differential equations,

$$
\begin{aligned}
(a X)_{, t} & =-\frac{\alpha Y}{r}+(\alpha Y)_{, r} \\
(a Y)_{, t} & =\frac{\alpha X}{r}+(\alpha X)_{, r} \\
\left(\frac{m}{r}\right)_{, r}+\frac{m}{r^{2}} & =\frac{1}{r}\left(X^{2}+Y^{2}\right) \\
\frac{(a \alpha)_{, r}}{a \alpha} & =\frac{2 a^{2}}{r}\left(X^{2}+Y^{2}\right) .
\end{aligned}
$$

The first two equations are equivalent to the Klein-Gordon equation in curved spacetime, and the last two are the Einstein equations determining the metric coefficients $a$ and $\alpha$ as functions of the energy density of the scalar field, which is proportional to $X^{2}+Y^{2}$.

Note that the form of these equations is invariant under the transformation

$$
\alpha(r, t) \rightarrow \alpha(r, t) \sigma(t) \quad d t \rightarrow d t / \sigma(t)
$$

This corresponds to the fact that the $t$ coordinate is not completely fixed by the form of (3). The degree of freedom inherent in $\sigma(t)$ can be eliminated by fixing the function $\alpha(r, t)$ at one value of $r$. In this paper we make the choice $\alpha(r=0, t)=1$ for all $t$, so that the $t$ coordinate measures proper time of an observer at the origin.

For near critical solutions, there is a sequence of echoing oscillations, each shorter than the previous one by a scaling factor of around 30. For supercritical solutions the sequence ends with horizon formation; for subcritical solutions the echo sequence dies out. For a perfectly critical solution, the solution studied by Gundlach [], the echoing would go through an infinite number of cycles. In all 
cases, the duration of proper time is finite, and is not highly sensitive to the way the sequence ends. From computational results with many echoes, then, the time $t_{*}$ for the end of the sequence can be estimated accurately. We adjust our time coordinate, so that $t_{*}=0$. That is, our zero of $t$ is chosen to be the endpoint of echoing.

Having fixed the zero and the scale of our $t$ coordinate, we next define the logarithmic coordinates

$$
\rho \equiv \ln r \quad \tau \equiv \ln (-t) .
$$

Notice that our choice of $\alpha$, and hence of $t$, differs from that of Choptuik [1] [he chooses $\alpha(r=\infty, t)=1$ ] but $\rho, \tau$ defined in (11) agree with Choptuik's logarithmic coordinates $\rho, \tau$.

Figure 1 shows a series of snapshots of the scalar field variable $X$ as a function of $\rho$ taken at various late times $\tau$. These are data made available by Choptuik on the internet from his numerical evolutions. In each of the plots we can distinguish two regions. On the left of the plots there is a "quiescent" region in which the solution dies off; on the right is an "oscillatory" region in which the solution has a sinusoidal appearance. We call the moving boundary between the two regions the "transition edge."
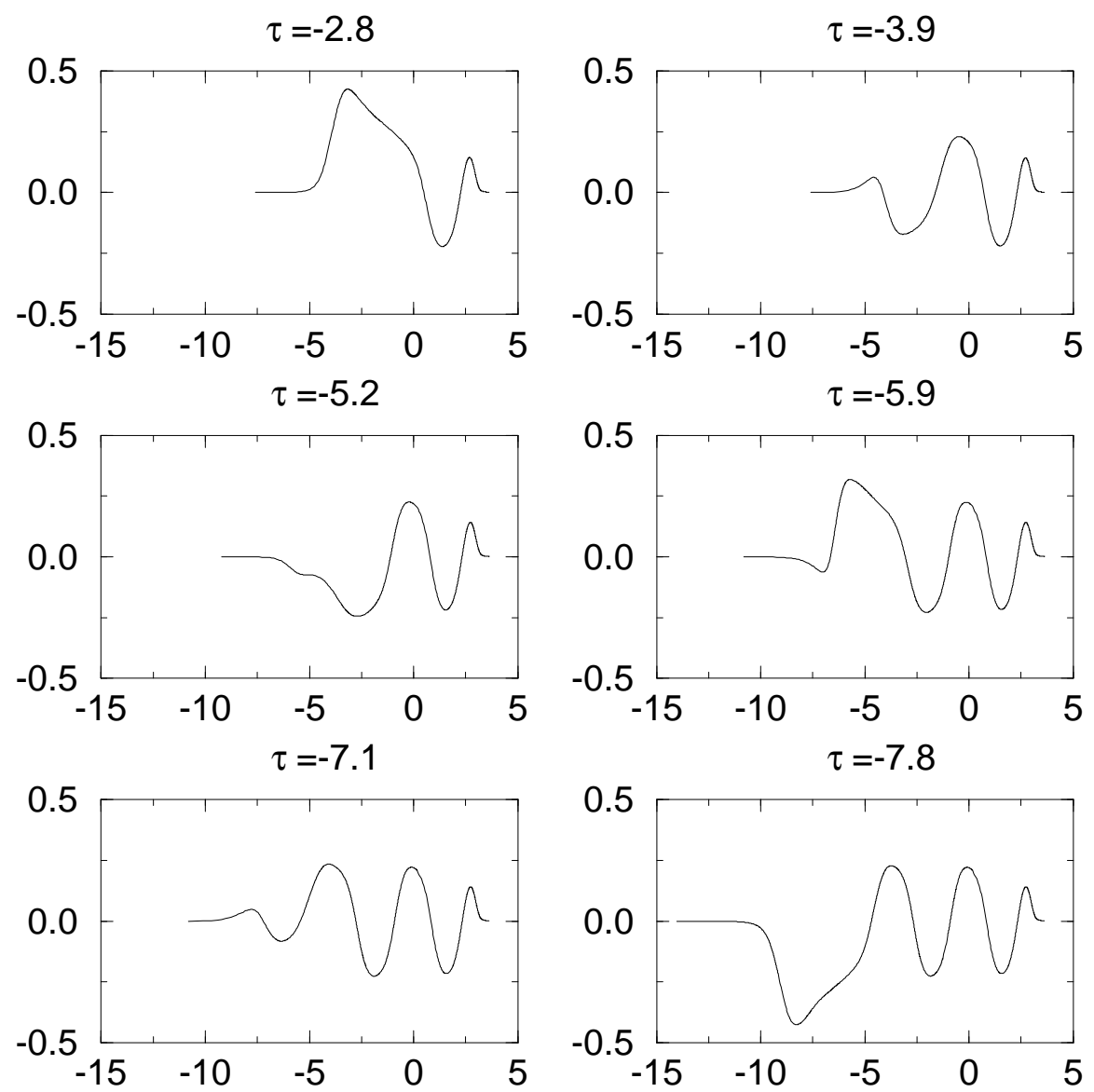

FIG. 1. The Choptuik numerical results for the scalar field variable $X$ as a function of the radial coordinate $\rho$, at different times $\tau$.

"Echoing" of the solution is the property that the pattern of $X(r, t)$ repeats itself on a changed radial and time scale according to

$$
X(r, t)=X\left(e^{\Delta} r, e^{\Delta} t\right),
$$

or equivalently

$$
X(\rho, \tau)=X(\rho+\Delta, \tau+\Delta)
$$


If $\Delta$ were a continuous variable, this property would tell us that $X$ is "self similar," i.e., a function only of $r / t$. But equations (12) and (13) are valid only for a fixed numerical value for $\Delta$, approximately 3.4 (a value of $3.4439 \pm 0.0004$ according to Ref. [7). For this reason the echoing property has been referred to as a "discrete self similarity."

Figure 2 shows the behavior of other variables of the problem, as functions of $\rho$ at a fixed $\tau$.
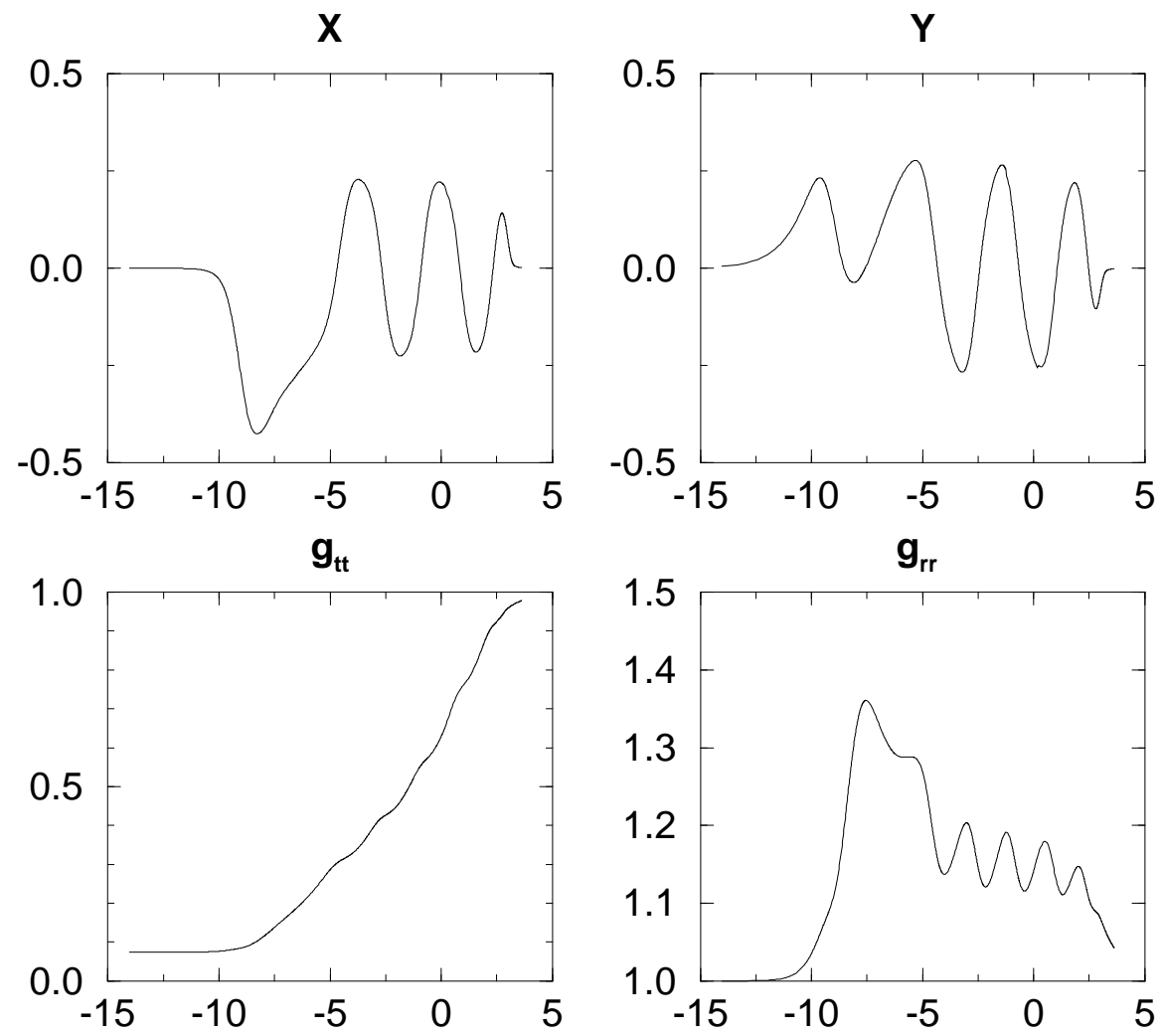

FIG. 2. The Choptuik numerical results for scalar field and metric variables, as a function of $\rho$, all at time $\tau=-7.87488$.

The scalar field variables $X$ and $Y$ have the appearance of being sinusoids approximately 150 degrees out of phase. Choptuik [8] has shown that it is useful to view the relation of $X$ and $Y$ in phase space plots, as in Fig. 3.

The elliptical portion of the plot corresponds to the oscillatory region of the solution. The transition region appears as the vertical spike which connects the elliptical windings to the origin. As radius increases, the windings decrease in size and the curve connects to the origin at a point representing spatial infinity in the numerical solutions. In a truly critical solution there would be an infinite number of windings and the connection with spatial infinity would be absent. 

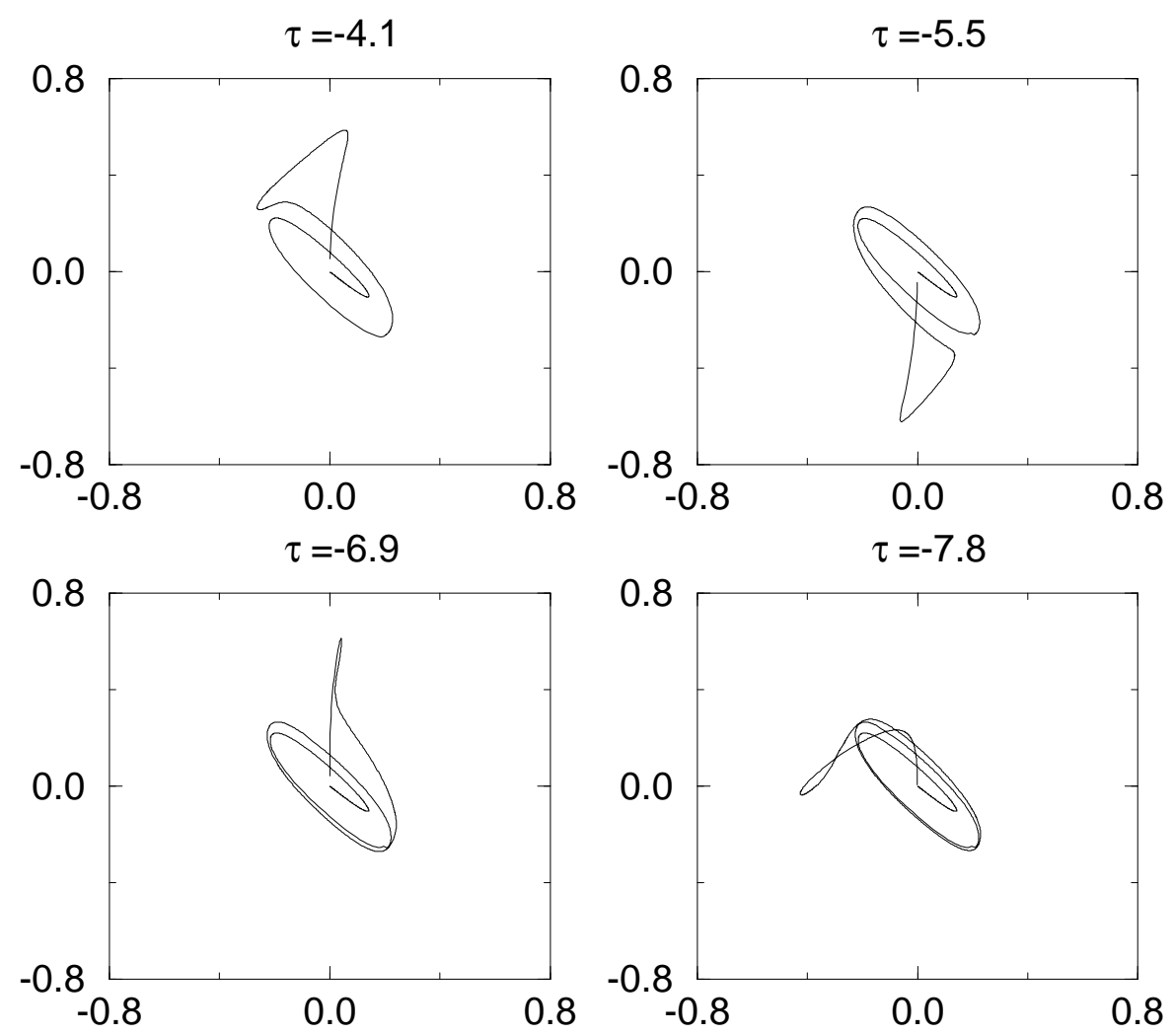

FIG. 3. The Choptuik numerical results for the relationship of the phase space variables $X$ and $Y$. Each plot corresponds to a single moment of $\tau$ and the full range of radii. The elliptical winding spiralling into the origin corresponds to radius growing large; the vertical spike approaching the origin corresponds to radius going to zero.

The qualitative features of $\alpha$, in Fig. 2, can be understood from (9). In this equation $\alpha_{, \rho}$ is "fed" by the source term $a^{2}\left(X^{2}+Y^{2}\right)$ which at each instant of time is a quantity bounded between zero and a value less than one. To get a rough understanding of the behavior of $\alpha$ we can take the source term as approximately constant and then the solution for $\alpha$ is

$$
\alpha \sim \mathcal{F}(\tau) e^{\kappa \rho},
$$

where $k$ is a constant related to the average value of $a^{2}\left(X^{2}+Y^{2}\right)$ and $\mathcal{F}$ is a function of time.

In view of the echoing, it is worthwhile to rewrite the equations in term of a new variable $\mu=\tau-\rho$. 


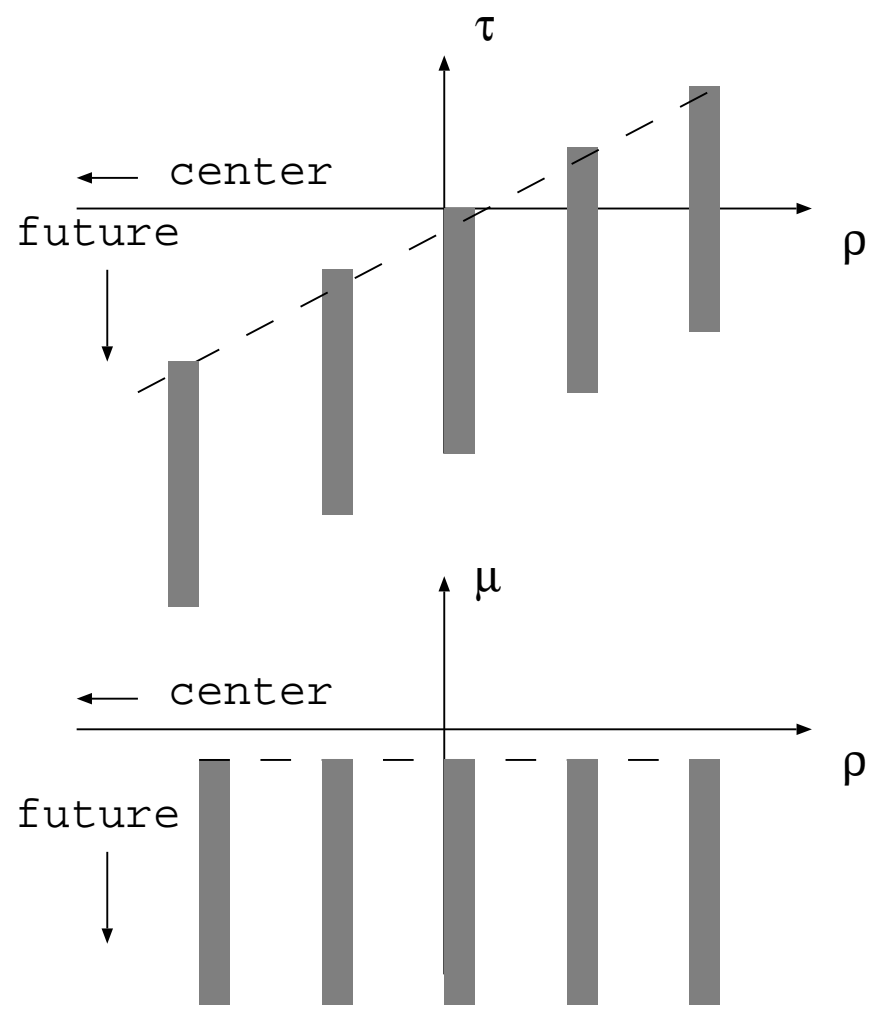

FIG. 4. Relationship of $\tau, \rho$ and $\mu, \rho$ coordinates. The dark bars represent the peaks of the $X$ oscillations; the dashed line shows the location of the transition edge. In both coordinate systems the region well above the transition edge is the "quiescent" region, and the region well below the edge is the "oscillatory" region.

Figure 4 shows schematically the location of the quiescent and oscillatory regions and of the transition edge, in terms of the $\tau, \rho$ and $\tau, \mu$ coordinates. In terms of these last coordinates, the Einstein-scalar equations are,

$$
\begin{aligned}
-e^{-\mu}(a X)_{, \mu} & =-\alpha Y+(\alpha Y)_{, \rho}-(\alpha Y)_{, \mu}, \\
-e^{-\mu}(a Y)_{, \mu} & =\alpha X+(\alpha X)_{, \rho}-(\alpha X)_{, \mu} \\
\left(\frac{m}{r}\right)_{, \rho}-\left(\frac{m}{r}\right)_{, \mu}+\frac{m}{r} & =X^{2}+Y^{2} \\
(\ln (a \alpha))_{, \rho}-(\ln (a \alpha))_{, \mu} & =2 a^{2}\left(X^{2}+Y^{2}\right) .
\end{aligned}
$$

As an example of the usefulness of the $\mu$ coordinate, we now go back to the behavior of $\alpha$ in (14) and consider the nature of $\mathcal{F}(\tau)$. At any $\tau$ the change in the "source" $a^{2}\left(X^{2}+Y^{2}\right)$ becomes sizeable at the transition edge $\mu \approx 0$. This tells us that at different times the characteristic rise of $\alpha$ as a function of $\rho$ will always occur around $\mu \approx 0$. For this to be true, and for $\alpha$ to have the form $e^{k \rho}$ at any one $\tau$, requires that the dependence of $\alpha$ on $\tau$ and $\rho$ have the form

$$
\alpha \sim e^{-\kappa \mu}
$$

in the oscillatory region, i.e., for $\mu \ll-1$. This approximation gives $\alpha$ a self-similar form, and is too crude, since the exact location at which $X, Y$ start to be large is not really $\mu=0$ but (since the solutions only have a discrete self similarity) oscillates in $\tau$. A better approximation which takes this oscillation into account, is

$$
\alpha=A(\tau) e^{-\kappa \mu},
$$

where $A(\tau)$ is a bounded, oscillatory function. 


\section{APPROXIMATE SOLUTIONS}

\section{A. Oscillatory region $\mu<<-1$}

We make the ansatz (motivated by the results in Figs. 1 and 2 ) that the $\mu$-derivative terms on the right in equations (15) and (16) are to be ignored in comparison with the $e^{-\mu} \partial_{\mu}$ derivatives on the left. We also introduce the variable

$$
u \equiv t / r=-e^{\mu}
$$

in terms of which the equations take the form

$$
\begin{aligned}
& (a X)_{, u}=-\alpha Y+(\alpha Y)_{, \rho} \\
& (a Y)_{, u}=\alpha X+(\alpha X)_{, \rho} .
\end{aligned}
$$

If we treat $a$ and $\alpha$ as constant, the equations admit the solution

$$
\begin{aligned}
& Y=A \sin [\omega(\rho-\alpha u)] \\
& X=A \sin [\omega(\rho-\alpha u)-\delta]
\end{aligned}
$$

with,

$$
\begin{aligned}
1+\frac{1}{\omega^{2}} & =a^{2} \\
\cos \delta & =-\frac{1}{a} \\
\sin \delta & =\frac{1}{\omega a} .
\end{aligned}
$$

If one fits this solution to the Choptuik's data in Figs. 1 and 2 , one finds that $\delta \approx 150$ degrees. The ratio of side lengths of the phase space ellipse is given by $\tan (\delta / 2)$, which yields a value of approximately 4, agreeing with the data due to Choptuik exhibited in Fig. 3.

Since we have assumed that $a$ and $\alpha$ are constant, we have in effect been considering the spacetime to be a flat background. This suggests that we should be able to find similar behavior in a solution to the scalar field wave equation in Minkowski spacetime. In this case $\phi$ can always be expressed, in terms of arbitrary functions $f, g$, as

$$
\phi=\frac{f(t-r)+g(t+r)}{r} .
$$

In terms of $f$ and $g$, Choptuik's phase space variables $X, Y$ are

$$
\begin{aligned}
& Y=f^{\prime}(t-r)+g^{\prime}(t+r) \\
& X=-\frac{1}{r}(f(t-r)+g(t+r))-f^{\prime}(t-r)+g^{\prime}(t+r) .
\end{aligned}
$$

To match a solution of this form to that of (24), (25) we take $f$ and $g$ to have forms chosen to give good agreement with (24), (25) for $\mu \ll-1$;

$$
\begin{aligned}
& f(\eta)=A \frac{1+k}{2} \frac{\eta}{1+\omega^{2}}[\sin (\omega \ln \beta \eta)-\omega \cos (\omega \ln \beta \eta)] \\
& g(\eta)=A \frac{1-k}{2} \frac{\eta}{1+\omega^{2}}[\sin (\omega \ln \beta \eta)-\omega \cos (\omega \ln \beta \eta)] .
\end{aligned}
$$

Here $A$ and $\beta$ are adjustable constants and $k^{2}=1+1 / \omega^{2}$. The agreement of this solution with the numerical results of Choptuik is most impressive in a phase-space plot, as in Fig. 5, where we have used the values $A=0.24, \omega=1.85, k=1.137$ in the flat spacetime solution. (The value of $\beta$ does not influence the appearance of the solution in this plot.) 


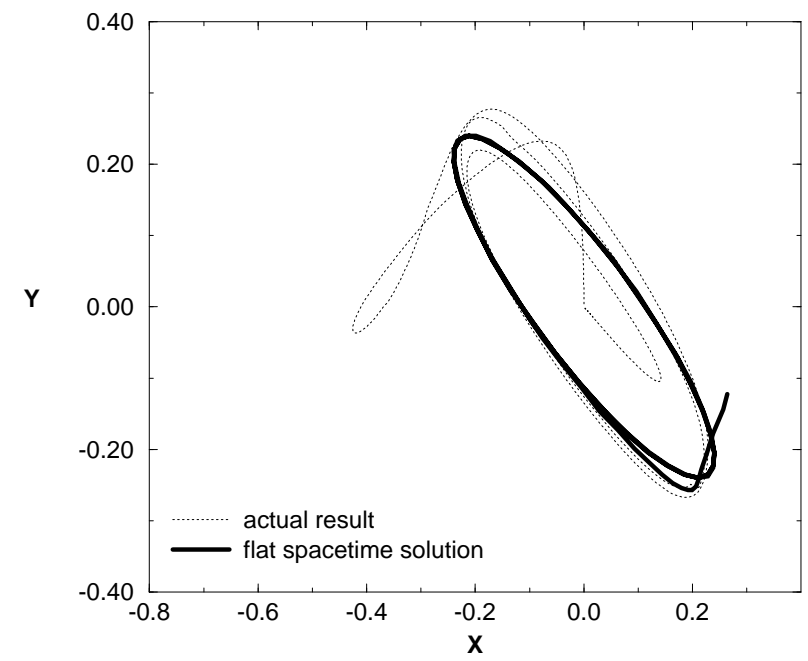

FIG. 5. A phase-space plot comparing Choptuik's numerical results with a flat spacetime solution at $\tau=-7.87488$. The flat spacetime solution corresponds to parameters $\omega=1.85$ and $A=0.24$.

The light dotted curve is Choptuik's result for $\tau=-7.87488$ and the thick curve is the flat spacetime solution. The thick curve, of course, lacks the vertical spike representing the transition edge. For the flat spacetime solution there is no transition front. The solution in $(32),(33)$ is a valid solution except at $t+r=0$ or $t-r=0$ (and is shown in Fig. 5 only for $t-r<0$ and $t+r>0$ ). It can agree with the Choptuik numerical results only in the region in which the approximations (22), (23) apply, i.e., it can agree only in the oscillatory region.

The flat spacetime fit of eqs. (32),(33), is plotted and compared to the numerical results of Choptuik in Figs. 6a,b. The agreement is remarkable in the oscillatory region, except at large $\rho$ (where Choptuik's results deviate from the ideal critical solution). The flat spacetime solution stops being reasonably accurate, of course, near the transition front, and deviates wildly from Choptuik's results in the quiescent region.

Despite the impressive agreement (in the appropriate region) of the simple solution above with the numerical results, the solution is less than ideal. For one thing it does not explain the gradual decrease in the amplitude of the oscillations seen in the numerical results. More worrisome is the question whether it is justified to treat $\alpha$ as constant when Fig. 2 shows it to be increasing exponentially. An improved approximation scheme which deals with such issues is possible, but is not central to our main point of this paper. 

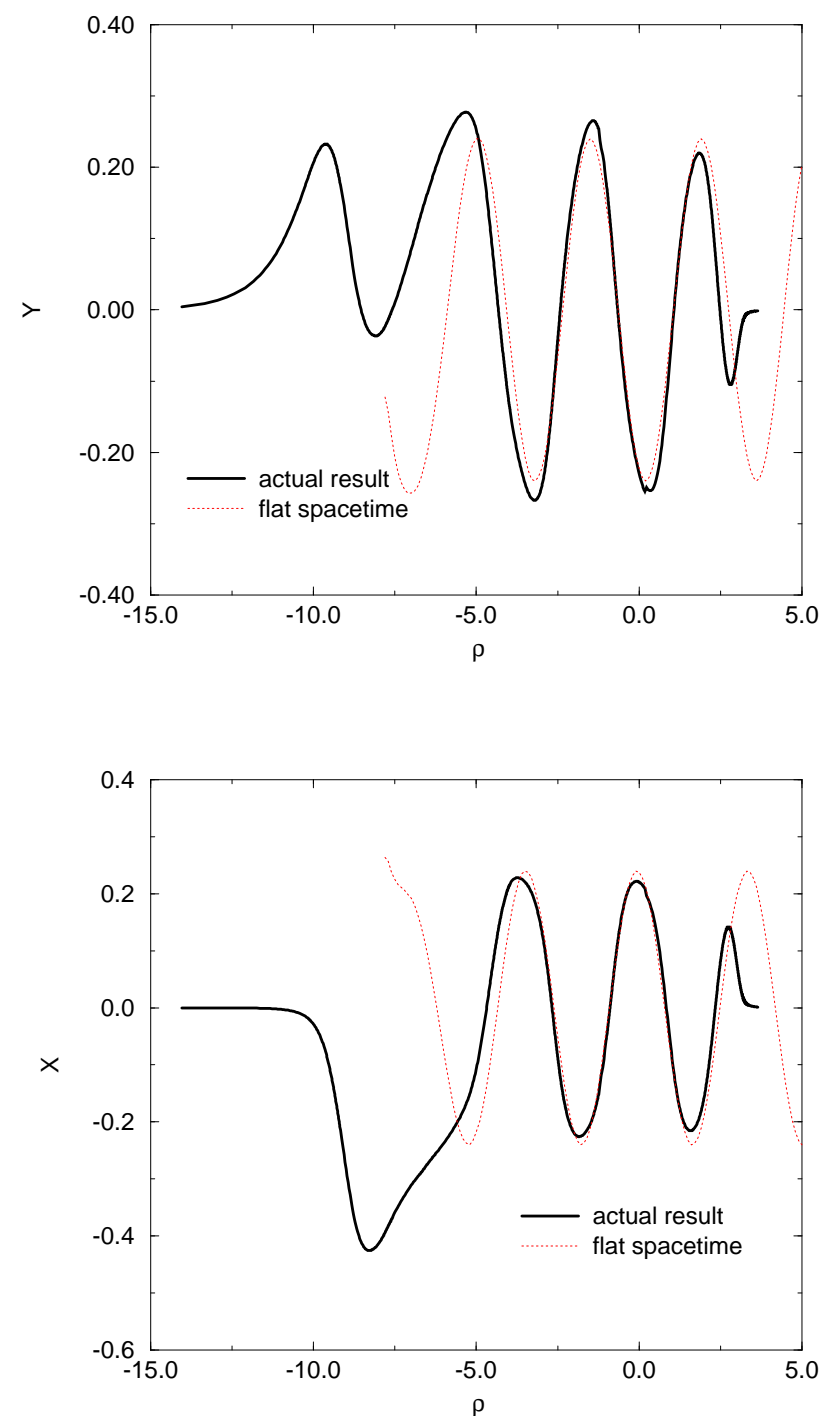

FIG. 6. The solutions $X(\rho), Y(\rho)$ found by Choptuik for $\tau=-7.87488$ are compared to the flat spacetime solutions corresponding to parameters $\omega=1.85, A=0.24, \beta=0.35$

\section{B. Quiescent region $\mu>>-1$}

In this region we drop the terms involving $e^{-\mu} \partial_{\mu}$ on the left of (15) and (16), and keep the $\partial_{\mu}$ terms. We make the convenient definitions $x \equiv \alpha X$ and $y \equiv \alpha Y$ so that the equations for $X$ and $Y$ (with the $e^{-\mu} \partial_{\mu}$ terms dropped) read,

$$
\begin{aligned}
y_{, \rho}-y_{, \mu} & =y \\
x_{, \rho}-x_{, \mu} & =-x .
\end{aligned}
$$

The general solution to these equations can immediately be written as

$$
y=e^{(-\mu+\rho) / 2} F(\tau) \quad x=e^{(\mu-\rho) / 2} G(\tau),
$$

in which $F$ and $G$ are arbitrary functions. At $\mu=0$ these solutions become

$$
y=e^{\rho / 2} F(\rho) \quad x=e^{-\rho / 2} G(\rho) .
$$



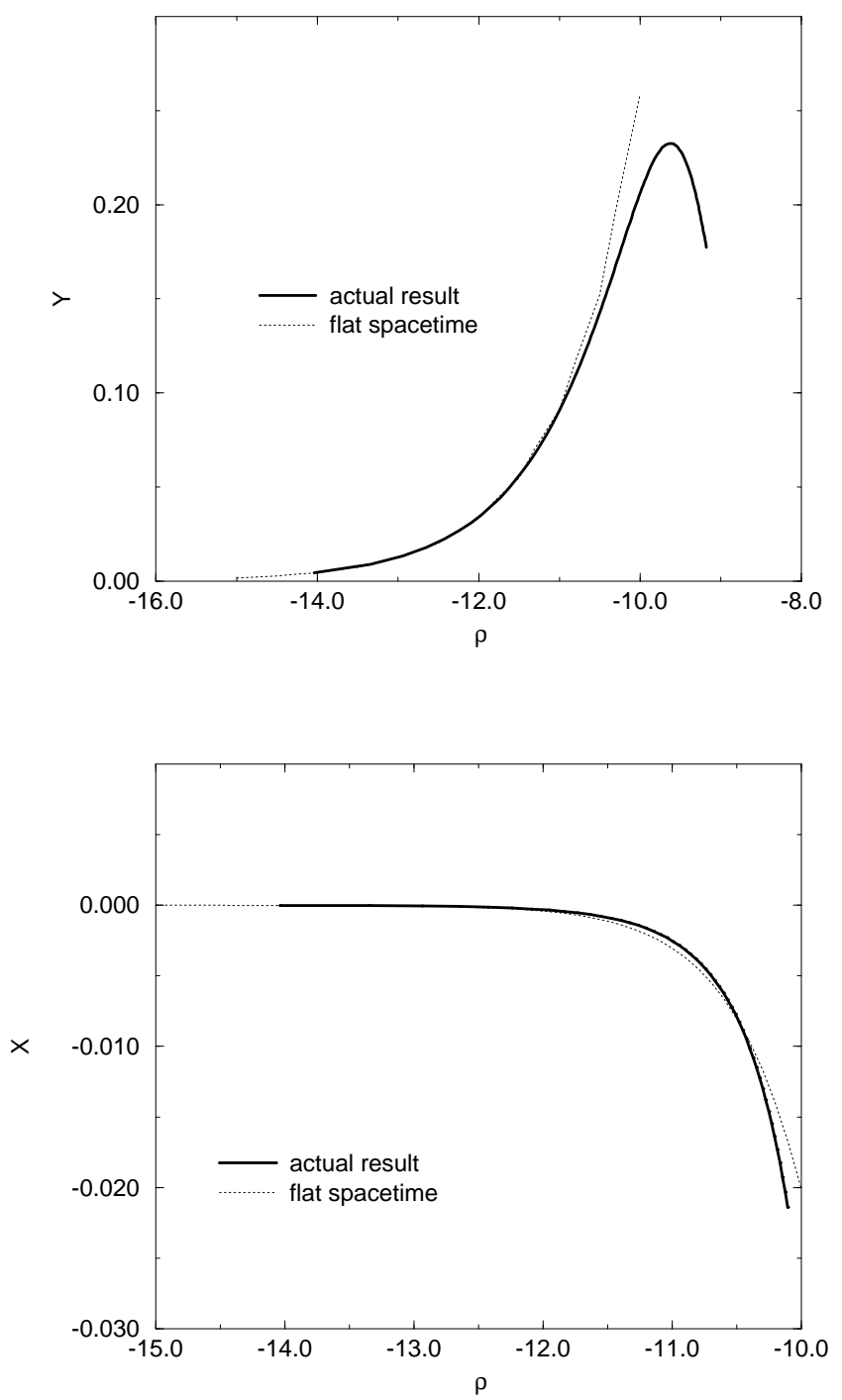

FIG. 7. The solutions $X(\rho), Y(\rho)$ found by Choptuik for $\tau=-7.87488$ are compared to the quiescent region flat spacetime solutions. Parameters $\mathcal{A}=0.324, \bar{\delta}=0.372$, have been used in the flat spacetime solution given in the text.

But at $\mu=0$, the solution is bounded as $\rho \rightarrow-\infty$, so $F(\xi)$ and $G(\xi)$ must respectively have the form $e^{-\xi / 2} \tilde{F}(\xi), e^{\xi / 2} \tilde{G}(\xi)$, where $\tilde{F}(\xi), \tilde{G}(\xi)$ are bounded as $\xi \rightarrow-\infty$. In the quiescent region, then, $x$ and $y$ have the form

$$
\begin{aligned}
& y=e^{\rho-\tau} \tilde{F}(\tau) \\
& x=e^{\tau-\rho} \tilde{G}(\tau) .
\end{aligned}
$$

At constant $\tau$, in the quiescent region, the scalar field variables vanish as $\rho \rightarrow-\infty$. This is satisfied by the above form for $y$, but for $x$ not to diverge, $\tilde{G}$ must be zero. This "first approximation" to $x$ and $y$ shows why $X / Y \rightarrow 0$ as $\rho \rightarrow-\infty$, and why the phase space plots have vertical spikes corresponding to the $\rho \rightarrow-\infty$ end of the plot. We can find a better solution for $x$ by substituting the above solution for $y$ into the left hand side of (16), and by treating $a$ and $\alpha$ as constants. This leads to

$$
x=e^{-2 \mu} \tilde{H}(\mu+\rho)
$$

where $\tilde{H}$ is given by 


$$
\tilde{H}(\xi)=-\frac{a}{3 \alpha}\left[\tilde{F}^{\prime}(\xi)-\tilde{F}(\xi)\right],
$$

From the results of Choptuik, such as those shown in Figs. 1 and 2, it is clear that the solutions in the quiescent region oscillate in $\tau$, with a period of $2 \pi / \omega$. The function $\tilde{F}$, then, must have this periodic property.

In the above, we have considered $a$ and $\alpha$ to be constant, so the solutions in (38) and (40), must be flat spacetime solutions. To see this explicitly, we use the flat spacetime forms of the solution in (30) and (31). Since $Y$ is finite as $r \rightarrow 0$ we must have $f(\eta)=-g(\eta)$. In the limit of small $r$ this gives us

$$
Y=-2 r f^{\prime \prime}(t)+\mathcal{O}\left(r^{2}\right) .
$$

By comparison with (38) we have that $f^{\prime \prime}(t)=-\tilde{F}(\tau) / 2 t$, where $\tilde{F}$ is a periodic function with period $2 \pi / \omega$. We illustrate the agreement of the Choptuik results with such a flat spacetime solution, by choosing

$$
f(\eta)=\mathcal{A} \eta \ln \eta \sin (\omega \ln \eta+\bar{\delta}) .
$$

Figure 7 shows the agreement, in the quiescent region, between the Choptuik results and the flat spacetime results of (15), (16), (38), at $\tau=-7.87488$. In (38) the parameter values $\mathcal{A}=0.324, \bar{\delta}=$ 0.372 , have been used.

\section{DISCUSSION}

In the previous section we introduced two approximate solutions that describe, with reasonable accuracy, the behavior of the Choptuik spacetime and we showed that these solutions were equivalent to flat spacetime solutions. Neither of these flat spacetime solutions, by itself, provides an acceptable solution in the full range of the spacetime manifold in which the Choptuik solution is found. From this point of view, the essential role of general relativity, and the nonlinearities it introduces into the equations, is to provide a match of the two solutions across the transition edge. We give here a very rough version of such a match. This is done to illustrate orders of magnitudes, but it is amusing (and coincidental) that this approximate match ends up giving a fairly accurate estimate of the period of the Choptuik echoing.

We know from the flat spacetime approximation in the echoing region that,

$$
1+\frac{1}{\omega^{2}}=a^{2} .
$$

We now adopt the viewpoint that $\omega$ is fixed by the way in which $a$ increases across the transition edge. The requirement of elementary flatness fixes $a$ to be unity at at $r=0$. Since $X$ and $Y$ are small in the quiescent region, we see from (8), here rewritten as

$$
\left(\frac{m}{r}\right)_{, \rho}+\frac{m}{r}=X^{2}+Y^{2}
$$

that $m / r$ must remain very small in the quiescent region, since $X$ and $Y$ are small. It follows that $a$ must be very close to unity in the quiescent region, and this is verified by Choptuik's results in Fig. 2. Equation (45) also allows us to make a crude estimate of how $a$ increases across the transition edge. From (38) the discussion in the previous section we can approximate $X \approx 0$ and (with $\alpha$ taken to be approximately unity) $Y \approx e^{-\mu} \tilde{F}(\tau)$. This approximation in (45) gives us

$$
\left(\frac{m}{r}\right)_{, \rho}+\frac{m}{r}=e^{2 \rho} e^{-2 \tau} \tilde{F}^{2}(\tau) .
$$

The initial condition on the solution is that $m / r=0$ at the origin $(\rho=-\infty)$, so that the solution everywhere in the quiescent region is approximately

$$
\frac{m}{r}=\frac{1}{3} \tilde{F}^{2}(\tau) e^{-2 \mu} .
$$


We now assume that this solution gives a roughly correct answer at the transition edge, $\mu=0$. Since $\tilde{F}$ is an oscillating function, $m / r$ also oscillates (see Fig. 2), and a representative value of $m / r$ is given by taking an average:

$$
<\frac{m}{r}>\approx \frac{1}{3}<\tilde{F}^{2}>
$$

We now argue that $\tilde{F}$ must be of order unity. The transition edge is defined as the location at which $X$ and $Y$ grow to order unity. If this is to be at $\mu=0$ then, from (38) it is clear that $\tilde{F}$ must be $\sim 1$. If, in fact, we take $\tilde{F}$ to be a sinusoidal oscillation of amplitude unity, then $\left\langle\tilde{F}^{2}>\approx 1 / 2\right.$, and we have from (48) that $\langle m / r\rangle \approx 1 / 6$.

As a consequence,

$$
a^{2} \sim 1+<\frac{2 m}{r}>\sim \frac{4}{3}
$$

which together with (44) implies,

$$
\omega \sim \sqrt{3}, \quad \Delta \sim \frac{2 \pi}{\sqrt{3}} \sim 3.6
$$

Of course, we could equally well have concluded, for example, that $\langle m / r>\approx 0.1$ and $\Delta \sim 2.8$. Our argument can only establish that $\Delta$ is of order unity. But this is enough to support our suggestion that it is the matching provided by the nonlinearities that picks out the form and the period of the Choptuik solution as the only one that can bridge the two approximately flat-spacetime solutions.

\section{ACKNOWLEDGMENTS}

We wish to thank Jürgen Ehlers, Bernd Schmidt and the Max Planck Institüt für Astrophysik at Garching for hospitality and support. This work was supported in part by grants NSF-PHY-9423950, NSF-PHY-9396246, NSF-PHY-92-07225, NSF-PHY-95-07719, research funds of the Pennsylvania State University, the University of Utah, the Eberly Family research fund at PSU and PSU's Office for Minority Faculty development. JP acknowledges support of the Alfred P. Sloan foundation through a fellowship.

[1] M. Choptuik, Phys. Rev. Lett. 70, 9 (1993).

[2] A. Abrahams, C. Evans, Phys. Rev. Lett. 70, 2980 (1993).

[3] J. Coleman, C. Evans, 72, 1842 (1994).

[4] D. Eardley, D. Hirschmann, "Universal Scaling and Echoing in Gravitational Collapse of a Complex Scalar Field", Preprint NSF-ITP-94-119, gr-qc@xxx.lanl.gov:/9412066 (1994).

[5] D. Maison "Non-Universality of Critical Behaviour in Spherically Symmetric Gravitational Collapse," preprint MPI-PhT/95-28, gr-qc@xxx.lanl.gov//9504008 (1995).

[6] C. Evans, private communication.

[7] C. Gundlach, "The Choptuik spacetime as an eigenvalue problem," preprint LAEFF-95/09, grqc@xxx.lanl.gov:/9507054 (1995).

[8] M. Choptuik, in "Approaches to numerical relativity", edited by R. D'Inverno, Cambridge University Press (1993). 